\title{
Conditioned Visual Flight Orientation in Drosophila: Dependence on Age, Practice, and Diet
}

\author{
Aike Guo, ${ }^{1}$ Liu Li, ${ }^{1}$ Xia Shou-zhen, ${ }^{1}$ Feng Chun-hua, ${ }^{1}$ Reinhard Wolf, ${ }^{2}$ and \\ Martin Heisenberg ${ }^{2,3}$ \\ ${ }^{1}$ Institute of Biophysics \\ Academia Sinica \\ Beijing 100101, China \\ ${ }^{2}$ Theodor-Boveri-Institut für Biowissenschaften \\ Lehrstuhl für Genetik, Am Hubland \\ D-97074 Würzburg, Germany
}

\section{Abstract}

Orientation preferences for visual patterns can be conditioned in tethered flies (Drosophila melanogaster) at the flight simulator. In a reversal conditioning procedure using heat as reinforcement, flies can be trained to successively prefer different flight orientations with respect to the patterns. As in many learning paradigms, conditioned responses are highly variable. Although during training most flies reliably avoid the heat and the corresponding flight orientations, in subsequent learning tests without heat some show no consistent preference for the permissive orientations. We have started to investigate the interindividual differences in learning performance and describe here three significant variables: the age of the animals, their experience in the flight simulator prior to the experiment, and the composition of the fly food. Flies learn more reliably at $3-4$ days than at 1-2 days of age but learning indices do not increase further in even older flies. Learning is improved if flies are allowed to become familiar with the flight simulator before the start of the conditioning procedure. Most important, poor nutrition causes complete amnesia within three or four generations. The reverse shift from poor to nutritious food restores learning ability with an even longer delay.

${ }^{3}$ Corresponding author.

\section{Introduction}

Behavioral modifications taken to represent learning and memory have been studied intensively at molecular and cellular levels (e.g., Tully et al. 1994; Kandel and Abel 1995). Less attention is paid to the respective behaviors that are modified. This limitation is paramount in studies in which the behavior is defined only operationally (e.g., shuttlebox, Morris water maze) or in which large groups of animals are used at one time (e.g., Quinn et al. 1974; reviewed in Heisenberg 1989). In Drosophila, the need for detailed behavioral analysis has been circumvented so far by the discovery of genetic variants that provided direct links between behavioral plasticity and biochemical processes such as cAMP signaling (for review, see Aceves-Pina et al. 1983; Dudai 1988; DeZazzo and Tully 1995). More recently, however, attempts have been made to relate these findings to neuronal pathways and centers in the brain (for review, see Davis 1993). This step may turn out to be much more difficult if the behaviors underlying the respective learning paradigms are poorly understood.

We studied heat-conditioned visual pattern discrimination of individual flies in the flight simulator (Wolf and Heisenberg 1991; Dill et al. 1993; Dill and Heisenberg 1995). This paradigm lends itself to a thorough behavioral analysis since visual flight orientation and in particular yaw torque in tethered flies have been studied extensively. The paradigm may be more demanding technically than others but the behavior under study is much better controlled than in freely walking or flying animals.

LEARNING \& MEMORY 3:49-59 (C) 1996 by Cold Spring Harbor Laboratory Press ISSN1072-0502/96 \$5.00

$$
\begin{array}{llllllllllllllll} 
& E & A & R & N & I & N & G & \begin{array}{l}
\boldsymbol{\chi} \\
49
\end{array} & M & E & M & O & R & Y
\end{array}
$$


It has been shown that this conditioning is an operant process (Wolf and Heisenberg 1991). Flies learn to use certain motor patterns generating yaw torque for stabilizing pattern orientations that are associated with the permissive temperature, and they maintain this behavior beyond the training period. Also, when confronted by heat they generate turning maneuvers to get back into a permissive orientation, but this response to heat seems not to be associated with the respective pattern orientation (Dill et al. 1995). The paradigm has been used extensively to study pattern recognition. The flies store the panorama as a memory template with fixed retinal coordinates (Dill et al. 1993). The template and the memory of the favorable flight orientations with respect to the pattern decay little during 20 hr (Dill 1995). Pattern recognition that is necessary for re-establishing the permissive flight orientations is a simple matching process in which the fly superimposes the actual image on the memory template. The process does not involve the analysis of geometrical shape (Dill and Heisenberg 1995).

In principle, the experiment is a single-fly learning test, because the same fly can be trained to change its preferred flight orientation (reversal conditioning; see Results). Yet the variability in the performance among individual flies is large and occasionally even whole populations of wildtype flies fail to learn. Large variability at the level of the individual is typical for learning tests in Drosophila (Quinn et al. 1974; Heisenberg et al. 1985; Heisenberg 1989). We therefore searched for uncontrolled variables. Here we describe several general properties of the conditioning of visual orientation in the flight simulator that account for some of the variability.

\section{Methods}

Unless stated otherwise, 3- to 7-day-old females of wild-type Berlin were used. Flies were grown on a standard cornmeal/molasses medium (see Table 1, Würzburg recipe) at $25^{\circ} \mathrm{C}$ and $60 \%$ humidity in a 16:8 hr light/dark cycle. To avoid overcrowding $\sim 100$ animals were transferred routinely to fresh $160-\mathrm{ml}$ food vials and allowed three days for egg laying.

The flight simulator and the conditioning procedure (Fig. 1a) have been described before (Heisenberg and Wolf 1984, 1988; Wolf and Heisenberg 1991). Briefly, the test fly is firmly at-
Table 1: Two Drosophila culture media

\begin{tabular}{lll}
\hline & $\begin{array}{l}\text { Würzburg } \\
\text { recipe }\end{array}$ & $\begin{array}{c}\text { Beijing } \\
\text { recipe }\end{array}$ \\
\hline Water & $1000 \mathrm{ml}$ & $1000 \mathrm{ml}$ \\
Cornmeal & $180 \mathrm{~g}$ & $84 \mathrm{~g}$ \\
Soybean & $10 \mathrm{~g}$ & - \\
Yeast & $18.5 \mathrm{~g}$ & $16.7 \mathrm{~g}$ \\
Agar & $7.5 \mathrm{~g}$ & $8.4 \mathrm{~g}$ \\
Molasses & $40 \mathrm{~g}$ & - \\
Syrup (sugar beet) & $40 \mathrm{~g}$ & - \\
Brown sugar & - & $108 \mathrm{~g}$ \\
Nipagin & $2.5 \mathrm{~g}$ & - \\
Propionic acid & - & $2.5 \mathrm{ml}$ \\
Benzoic acid & - & $0.63 \mathrm{~g}$ \\
\hline
\end{tabular}

tached by its thorax and head to a hook. It is surrounded by a translucent drum with visual patterns on its periphery. The fly's yaw torque is recorded and is transformed continuously and instantaneously into the negative angular velocity of the drum (negative visual feedback). The fly stabilizes the panorama and chooses its direction of flight with respect to the visual patterns. For conditioning, certain pattern orientations are associated with heat, i.e., heat is switched on whenever these patterns enter the frontal $90^{\circ}$ sector of the fly's visual field. Flies learn quickly to avoid pattern orientations combined with heat and retain the respective pattern preferences even after heat is switched off.

Performance indices ("preference index" before, "avoidance index" during, and "learning index" after the training) are calculated as $\mathrm{PI}=$ $\left(t_{C}-t_{H}\right) /\left(t_{C}+t_{H}\right)$, where $t_{C}$ is the time the fly is oriented toward a sector not associated with heat and $t_{H}$ is the time the fly is oriented toward a sector that, during training, is associated with heat. The only patterns used in this study were an upright and an inverted $T$ (total horizontal and vertical width of figures $40^{\circ}$, of bars $8^{\circ}$ ). Background luminance $I=400 \mathrm{~cd} / \mathrm{cm}^{2}$ in the experiments of Figures $2-7$ and $I=90 \mathrm{~cd} / \mathrm{cm}^{2}$ in the experiment of Figure 1. Data of Figures 1-4 were obtained in Würzburg, those of Figures 5-7 in Beijing. Error bars in Figures $1 \mathrm{~b}, 2,3$, and 5 are S.E.M.s. In most instances avoidance and learning indices are not corrected for the spontaneous pattern preferences of the individual flies. In Figures 6 and 7 in each generation the mean three preference, four avoidance, and five learning indices (see Fig. 5 for time

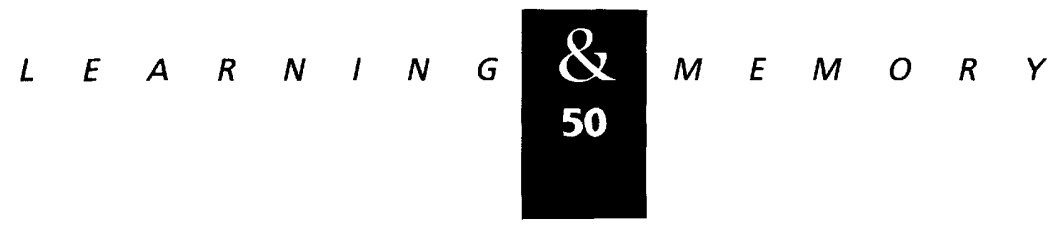




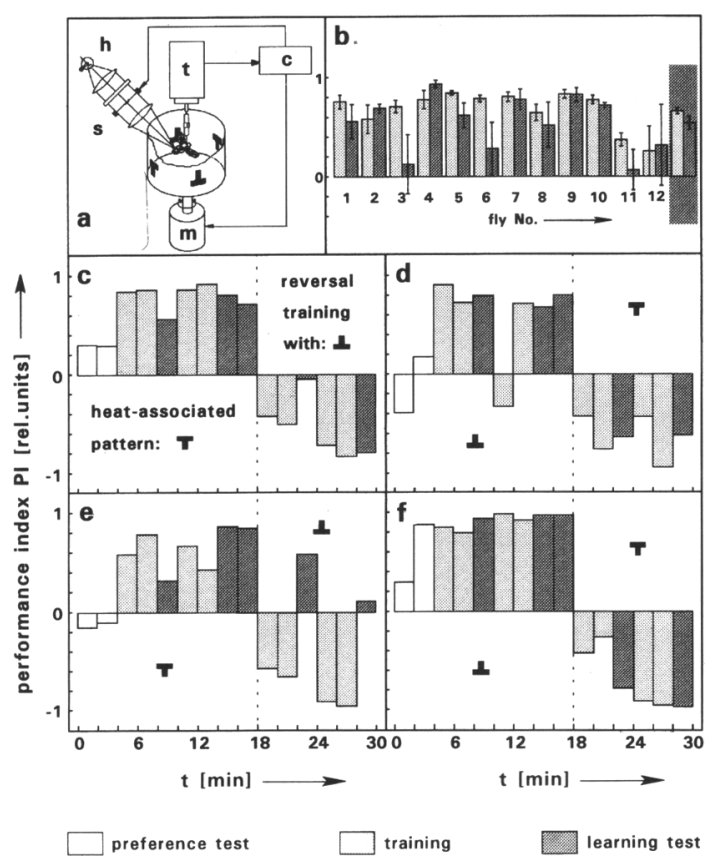

Figure 1: Reversal conditioning of 12 individual flies in the flight simulator. (a) Experimental set-up with flight simulator and heat lamp for reinforcement. The visual stimulus consists of two upright and two inverted T-shaped figures in the four quadrants of a brightly illuminated arena, with identical patterns facing each other. (c) Computer; (h) heat source; $(m)$ : motor; $(s)$ shutter; $(t)$ torque meter (see Methods for further details). (b) Mean avoidance (light gray) and learning indices (dark gray) of each fly. Only the first learning index after each training is used for averaging. At the far right the means of all 96 avoidance and 48 learning indices are shown on a shaded background. $(c-f)$ Performance of four individual flies. Flies are first tested for their spontaneous pattern preference $(2 \times 2 \mathrm{~min})$ and are subsequently conditioned with heat to avoid one of the two patterns. After two training and two test periods (dotted vertical line at $18 \mathrm{~min}$ ), the relation between pattern orientations and heat is reversed. Now, flies are heated whenever they assume flight orientations that previously had been "safe."

course of experiments) are averaged and the resulting preference index is subtracted from both the avoidance and learning index. S.E.M.s of the final indices are obtained by error propagation.

\section{Results}

\section{REVERSAL CONDITIONING OF SINGLE FLIES}

In the flight simulator with heat as negative reinforcer, individual flies can be conditioned quickly and efficiently to prefer certain flight orientations to others. To demonstrate this, a fly was first conditioned to avoid one of two pattern orientations, and subsequently the contingency between heat and pattern orientations was switched such that the fly had to avoid the previously preferred pattern orientations. Reversal conditioning experiments with 12 individual flies are shown in Figure 1. In Figure 1c, for instance, the fly was first conditioned to avoid orientations toward the upright $\mathrm{T}$, i.e., whenever an upright $\mathrm{T}$ entered the frontal $90^{\circ}$ sector (i.e., $\pm 45^{\circ}$ from the frontal midline), the heat was turned on. After $18 \mathrm{~min}$ the contingency between pattern orientations and reinforcer was inverted. All the previously "safe" orientations were now "forbidden" and vice versa. In the first training session after the switch (18-22 min) the fly may have been confused, as can be deduced from the low avoidance indices. The subsequent learning index (22-24 $\mathrm{min}$ ) was close to zero. Considering the very large learning index just before the reversal $(14-18 \mathrm{~min})$, the fly seemed to have learned already to give up the previous pattern preference. Avoidance indices during the second training period after the switch and in the following test were very high. In Figure 1f the fly developed a pattern preference for the upright $\mathrm{T}$ already before the reinforcement. Consequently, because the upright $T$ was first associated with nonheat, avoidance and test indices were extremely high. After the switch, when the preferred pattern orientation was associated with heat, the fly showed a particularly low first avoidance index but during that 4-min period it changed its preferred flight orientation and henceforth most of the time headed toward the inverted $T$.

Some flies in Figure 1b did not show a consistent performance after the switch, as if they were confused; others may have taken longer than the recording period to develop the new orientation preference, as suggested in Figure 1e. If flies continued to fly, the contingency between pattern orientation and temperature could be switched back and forth several times. Subsequent switches seemed not to be more (but also not less) confusing for the fly than the first one (no data shown). Unfortunately, few flies cooperated long enough to make such experiments feasible as the routine (but see the modified paradigm of A. Guo and K.G. Götz, in prep.). Nevertheless, the individual data as well as the means of the 12 experiments (Fig. 1b) indicate that in the flight simulator flies can adopt

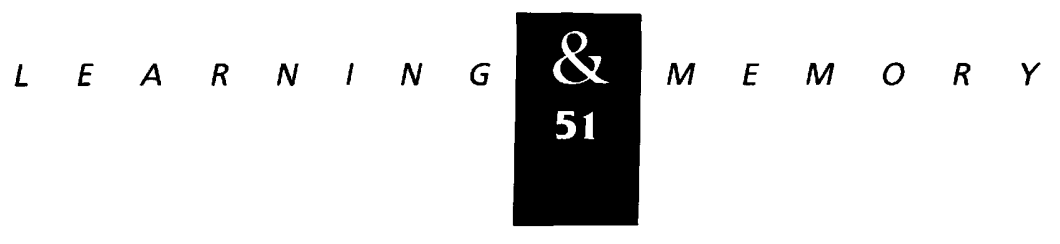


new preferred flight orientations repeatedly (for reversal conditioning in odor discrimination learning, see Quinn et al. 1974; in spatial learning, see Wustmann et al. 1996).

However, individual flies differ dramatically in their learning performance. In Figure 4 for each of 68 flies the learning indices of individual flies tested without reversal are averaged and plotted in a scatter diagram (the abscissa of the diagram will be explained below). Values range from $P I=-.38$ to $\mathrm{PI}=.93$ and are about normally distributed, with a slight accumulation of data at the upper end of the scale. Empirically, this variability has led to the routine of averaging about 20 single-fly experiments for a reliable mean learning index, amounting to a recording time of $6 \mathrm{hr}$. In order to understand and possibly reduce this broad distribution, we studied some of its causes.

\section{LEARNING PERFORMANCE INCREASES BETWEEN 2 AND 4 DAYS OF AGE}

First we investigated whether part of the interindividual variability is due to the differences in age. In our standard protocol age is not rigorously controlled. At the time of measurement flies are from 3 to 7 days old (Wolf and Heisenberg 1991). To test age dependence newly eclosed imagines were collected every $24 \mathrm{hr}$ and transferred to fresh culture vials. Ten hours later the "young" flies were glued to wire hooks and stored in small vials for 14 to $24 \mathrm{hr}$ until they were used in the experiment. The remaining flies were left on the standard food for an additional $48 \mathrm{hr}$ before being attached to the hooks ("old").

In Figure 2, the heat avoidance and learning indices of (a) 50 young and (b) 24 old flies are shown. As is apparent from the data, both groups of flies avoided the heat-associated sectors about equally well. After the first 4-min training period learning indices are small and not significant for both groups. In the learning tests following the second training period the old flies performed significantly better than the young ones. For the latter, none of the learning indices is significantly different from zero, whereas the corresponding values of the old animals are all significantly positive at the $5 \%$ level.

To test for the robustness of this effect the experiment has recently been repeated in Beijing (23 young and 20 old flies) with essentially the

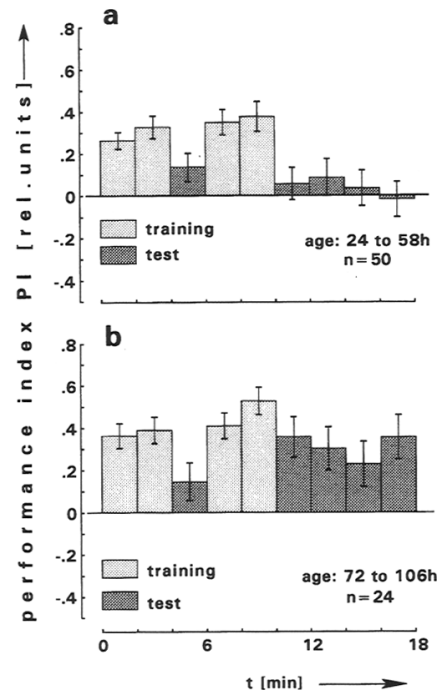

Figure 2: Age dependence of pattern avoidance conditioning. About half the flies are trained to avoid the upright $T$, the other half the inverted one. (a) Flies between 1 and 2.5 days of age avoid heat-associated flight orientations but show no learning. Even with 50 animals the first learning test is not significant at the $5 \%$ level. Compare, however, the just significant learning index of the first test in the identical experiment of Fig. 3a. (b) Flies between 3 and 4.5 days of age show significant learning indices after the second training period for each of the four learning indices $[P<0.05$; single-sample $t$-tests (Sachs 1992)]. Flies of the two ages differ significantly from each other $\left[P=0.0292 ; V_{(4,69)}=2.87\right.$; oneway ANOVA, multiple comparison test for repeated mesures]. Some of the flies in a are siblings of the flies in $b$, others were tested on the same days. Note that no pattern preference tests are performed before the conditioning experiment. (n) Number of flies.

same results. Again, in both groups the learning index after the first training was close to the 5\% level of significance, and the test values for the young animals after the second training were not significantly different from zero and were lower than those of the old flies (data not shown; but see Fig. $3 a$ for further data of young flies). We assume that a small part of the variability among the 3- to 7-day-old flies in the standard experiment is due to the age dependence of the learning indices.

We considered the possibility that young flies might be more vulnerable to cold anesthesia and the gluing procedure than old ones. In a control experiment, therefore, young and old flies were glued to the wires at the same early time and the old flies were stored with their hooks for the ap-

$$
\text { ….... }
$$


propriate time. Flies gained learning competence while they were aging irrespective of whether they carried a hook or not (data not shown).

\section{PRACTICE IN THE FLIGHT SIMULATOR IMPROVES SUBSEQUENT LEARNING}

Next we asked to what extent a fly's experience prior to the experiment would affect its learning performance. Some flies may never have tried to fly before being hooked to the torque meter; others may have practiced flight in the limited space of the food bottle. We allowed flies to fly in the flight simulator for $6 \mathrm{~min}$ prior to the experiment without heat reinforcement. During that phase the flies' spontaneous pattern preferences were recorded for three 2-min periods. (The PIs for pattern preference in Fig. 3b,d are not included in the figure because about half the flies were trained to avoid the upright $T$ and the rest to avoid the inverted $T$. See Methods for calculation of PIs.) The PI values during the subsequent heat conditioning and learning tests were compared with those of flies that started the experiment without prior practice. (Note the 4-min preference test in the experiment of Fig 1.)

Experience in the flight simulator prior to heat conditioning, indeed, causes a substantial improvement in the learning performance. In Fig. $3 \mathrm{a}, \mathrm{b}$ half of the 32 young flies are subjected to the 6-min practice in the flight simulator, the other half are not. The experienced animals in b learn well. Learning performance of the inexperienced flies (a) is small after the first and second training and vanishes completely in the subsequent three test intervals, in accordance with the results presented in Figure 2a. Note that even avoidance during the first $2 \mathrm{~min}$ of training is significantly lower than in the experienced flies. A less dramatic result is obtained with old flies (Fig. 3c,d). Here the difference between the experienced and unexperienced flies is in the same direction but is not significant.

This finding raises the question of what the fly learns during the pretraining period. Although we have no definitive answer we assume that it is the stabilization of the panorama. Support for this assumption is provided by the following observation. Some of the experiments were performed in a flight simulator in which the coupling coefficient (for review, see Heisenberg and Wolf 1993) be-

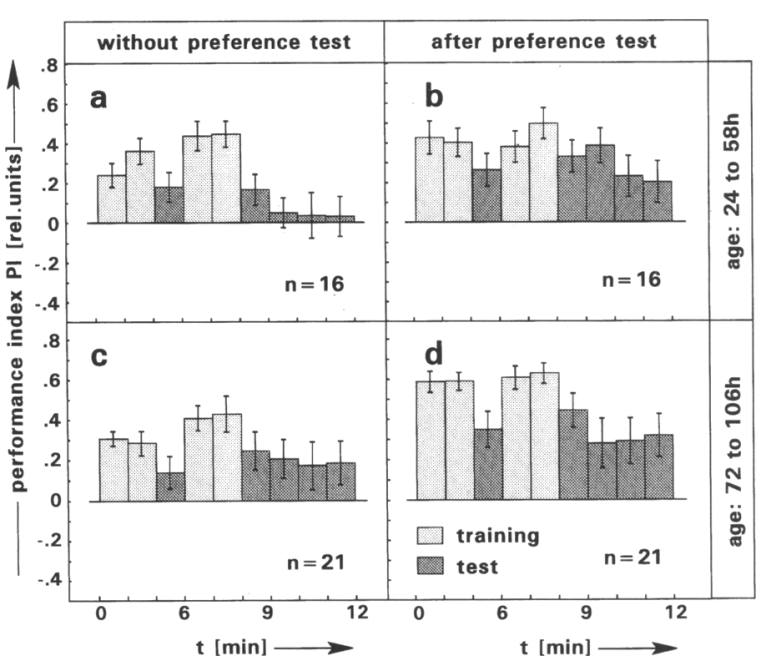

Figure 3: Influence of preference test on learning. Experimental conditions in a and $c$ are the same as in the experiment of Fig. 2. In $b$ and $d$ flies are tested for $6 \mathrm{~min}$ without reinforcement before the onset of the first training period. In the low-age group $(a, b)$ after the second training period the improvement due to the pretraining is significant at the $5 \%$ level $\left[P<0.04 ; V_{(4,27)}=2.91\right.$; oneway ANOVA, multiple comparison test for repeated measures]. In the older flies $(c, d)$ although suggestive due to the higher avoidance indices, the improvement after pretraining is not significant $[P<0.408$; $\left.V_{(4,37)}=1.02\right]$. (n) Number of flies.

tween yaw torque and angular velocity of the panorama was twice the normal value $\left[\mathrm{k}=-22^{\circ} / \mathrm{sec} /\right.$ $10^{-10} \mathrm{Nm}$ (instead of $\mathrm{k}=11^{\circ} / \mathrm{sec} / 10^{-10} \mathrm{Nm}$ )] This value is close to the upper limit of the range in which the fly can stabilize the panorama. We figured that the preference index for one or the other pattern can be high for a 2 -min interval only if the fly is able to stabilize the panorama well. On the other hand, the inverse is not the case: If a fly can stabilize its course in the flight simulator, the preference index must not necessarily be high. In Figure 4, therefore, for a large number of individual flies the largest of the three preference indices is plotted with its absolute value $\left(|\mathrm{PI}|_{\max }\right)$ against the mean learning index obtained from this animal. A substantial positive correation is observed, implying that flies with a strong pattern preference show good learning irrespective of the relation between the spontaneous and the enforced preference.

We interpret this to indicate that only a fly that can stabilize the panorama well and keeps steady pattern orientations is able to show its pat-

$$
\begin{array}{llllllllllllllll}
\hline & E & A & R & N & I & N & G & \begin{array}{llllllll}
\mathbf{X} \\
53
\end{array} & M & E & M & O & R & Y
\end{array}
$$




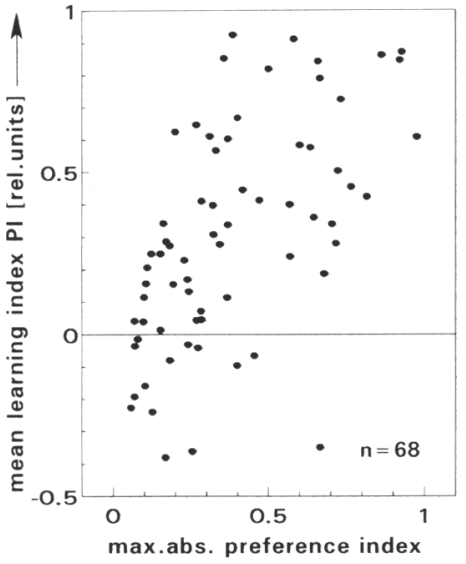

Figure 4: Scatter diagram of mean learning indices versus maximal absolute preference indices of individual flies. Experimental conditions as in Fig. 3d. For each fly all five learning indices are averaged and plotted on the ordinate. (The mean learning index is chosen to reduce the scatter in the data. If each 2-min interval is averaged over the 68 flies the five learning indices are not significantly different from each other. For comparison, see also the mean learning indices at different times in the experiments shown in Figs. 2 and 3). Prior to conditioning the spontaneous pattern preference is recorded in three 2-min intervals, and the largest absolute value of these three PIs is plotted on the abscissa as a measure of the fly's ability to stabilize the panorama. Correlation coefficient $r=0.62 \quad(9 \%$ confidence interval: $0.45<r<0.75$; for difference to zero: $P<0.0001$; Spearman Rank Correlation).

tern preferences and that what the fly learns during the pretraining is to stabilize the panorama. This view is further supported by an earlier finding of Dill (1995) showing that the steadiness of orientation increases continuously at the flight simulator irrespective of heat reinforcement.

To assess whether experience in free flight is important for the performance in the flight simulator, flies are kept in large flight cages before being tested at the torque meter. No significant improvement in learning indices is observed even for flies that have been chased around extensively in the cage (data not shown). Probably, the situation in the flight simulator is so artificial that experience in free flight is not very helpful. In summary, our data indicate that experience with the flight simulator does influence the subsequent conditioning experiment, but we have no direct evidence that general experience prior to the experiment contributes to the variability in learning indices.

\section{A FLY FOOD THAT SUPPORTS AVOIDANCE BUT NOT LEARNING}

Life in a food bottle changes dramatically during the few weeks of its maintenance. In a rich food medium, the first 100-200 larvae develop with unlimited supplies but for the later-hatching animals, nutrients soon get depleted and deteriorate: The concentration of excrements increases; fast-growing microorganisms may produce noxious substances; development of the larvae slows down; and a devastating competition starts among them. Also for the imagines the lack of food and the increasingly gluey consistency of the substrate make life exceedingly difficult. It has been shown that the conditions of larval growth have a profound effect on the size of the imaginal mushroom bodies (Heisenberg et al. 1995) and on other parts of the adult brain (M. Barth, H.V.B. Hirsch, I. Meinertzhagen and $M$. Heisenberg, in prep.).

It is difficult to assess the effect of larval growth conditions on the interindividual variability in learning performance. Inadvertently, however, we performed an experiment that bears on this question. The fly food regularly used in Beijing contains mainly carbohydrates and little organic nitrogen and phosphorous. It probably is close to a minimal medium (Table 1). Even in fresh cultures Iarval development was substantially retarded, taking 4 days longer than in Würzburg food.

Flies grown on Beijing food, nevertheless, had normal size and appeared healthy. Tethered flies flew readily and could keep stable orientations in the flight simulator. In visual pattern conditioning they were well able to avoid the heat during training. In the subsequent learning test, however they showed no trace of a preference for the "cold" pattern (Fig. 5). Obviously, the Beijing diet does not support visual learning at the flight simulator. Curiously, in fresh cultures of Beijing food, males were hyperactively courting females as if normal courtship suppression by mated females (Tompkins et al. 1983) did not work properly. This observation has not been followed up. Note that the learning deficit was not caused by contamination of the ingredients (herbicides, heavy metals, etc.) but by the recipe. We tested both recipes with ingredients from Beijing and Würzburg. The source of the materials was determined to be irrelevant. Nutritional requirements for learning have not been worked out yet. Only the role of the antifungal drugs (nipagin vs. propionic acid/ben-

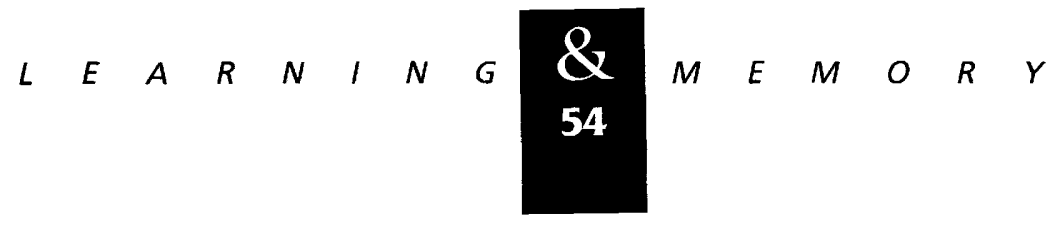




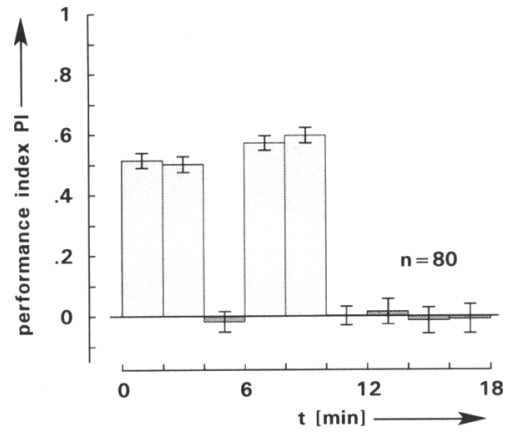

Figure 5: Mean avoidance and learning indices of 80 flies $(n)$ raised for more than five generations on Beijing food. Experimental conditions as in Fig. 3d. For comparison of Beijing and Würzburg food see Table 1. (Light gray bars) Training; (dark gray bars) learning test.

zoic acid; see Table 1) has been tested by exchanging these in the recipes. No effect on learning indices of the drugs at the indicated concentrations was apparent (S.Z. Xia, L. Liu, C.-H. Feng, and A.K. Guo, in prep.).

We wondered whether it was the actual nutritional state of the animal in the experiment or the chemical environment of larval development that determined the amnesia or the learning propensity. The answer is given in Figures 6 and 7 . Clearly, the immediate nutritional state had no measurable effect. When flies from cultures grown on Beijing food were transferred to the Würzburg diet right after eclosion from the pupal case (generation 0 ) and were tested 3 or 4 days later, they remained fully amnesic. The same was true for the opposite change: In flies grown on Würzburg food, Beijing food during adult life seemed to have no significant effect on learning performance.

\section{GRADUALLY INCREASING EFFECT OF THE DIET}

If it is not the actual nutritional state of the imago that matters, one is inclined to assume that it must be the embryonic and larval development that is channeled into different directions in the two chemical environments. This, however, seems not to be the whole story, either. Even in the first generation after the change to the new diet the effect is small. For stocks continuously grown on Beijing food it took $\sim 4-5$ generations of growth on Würzburg food until the flies had fully recovered from amnesia (Fig. 6). Inversely, the F1 of flies switched fom Würzburg to Beijing diet was only partially amnesic and it took again about three generations until learning ability was fully blocked (Fig. 7). Possibly, the deleterious effect of poor food makes itself felt faster than the beneficial influence of the nutritious medium. In the F1 generation, after a switch from Beijing to Würzburg food only about $25 \%$ of the final learning index was restored, whereas in the F1 after the opposite switch about $50 \%$ of the learning index was lost. Note that in Figure 6 all the data for the switch from Beijing to Würzburg food have been included. They derive from two experimental series performed several months apart. One is shown separately as the filled symbols in Figure 7 . In the latter figure the shift from Würzburg to Beijing food and the inverse shift were performed at the same time. In subsequent generations the days for experimentation diverged because of the different developmental times on the two foods. It was important to perform the two shifts simultaneously because occasionally we encountered periods of several weeks during which wild-type flies showed poor conditioning. Note that the diet seemed to have no substantial effect on heat avoidance (circles in Figs. 6 and 7).

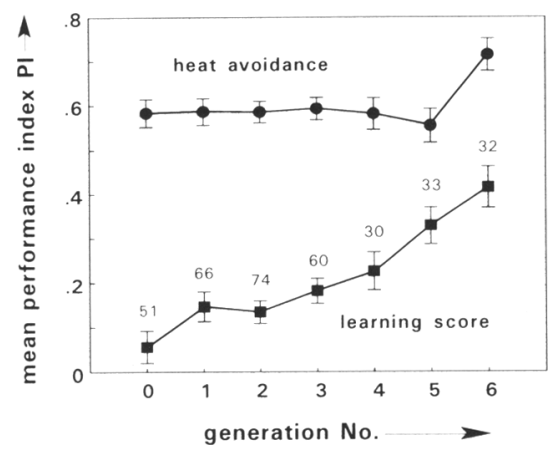

Figure 6: Increase of learning performance over seven consecutive generations of flies raised on Beijing food for more than 4 months prior to the experiment and shifted to Würzburg food at eclosion in generation 0 . Avoidance ( ) and learning indices ( $\mathbf{\square})$ are the means of the four (avoidance) and five (learning) indices recorded in the standard procotol (see, for instance, Fig. 5). Preference indices measured during $6 \mathrm{~min}$ before the training are subtracted. Note the exceptionally high avoidance index in generation 6, which raises the question of whether the increase in learning from generation 5 to generation 6 is real. This difference is statistically not significant $\left[P<0.1 ; t_{(63)}=1.4\right.$; unpaired $t$-test $]$. Numbers above the data points of learning indices refer to no. of flies tested in each generation. Error bars (S.E.M.S) are derived by error propagation from the respective S.E.M.S of the five learning and four avoidance indices.

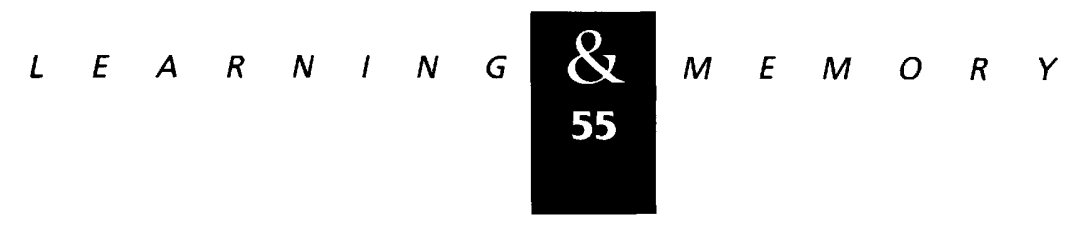




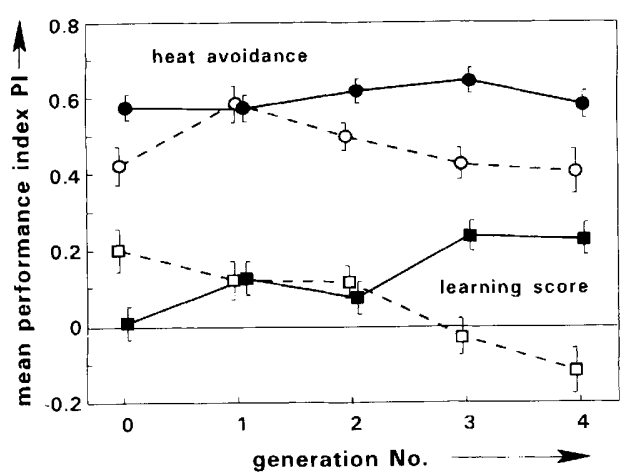

Figure 7: Two parallel cultures, one permanently grown on Beijing food $(\mathbf{D})$ the other on Würzburg food $(O, \square)$ are shifted at the same date to the opposite diet. The figure shows avoidance (circles) and learning indices (squares) for five consecutive generations. Twenty flies are averaged in each generation for the shift from Würzburg to Beijing food, 30 flies for the Beijing to Würzburg shift (29 flies in generation 0 ). Sequences of training and test periods are recorded as in Fig. 5 and averaged as explained in Fig. 6 . The data represented by the filled symbols are also part of the larger data set in Fig. 6. Error bars are obtained as described in the legend to Fig. 6.

\section{Discussion}

Single-fly learning tests are compromised by a large variance in learning indices because the behavior of the individual does not reliably reflect the group average. Understanding some of the causes of this variance is of practical and theoretical interest. It may help to reduce them and to explain their functional significance. The experiments presented here do not measure directly how much the variables studied contribute to this variance. The heterogeneity in age that we allow for in our standard protocol is likely to contribute to it. How much individual previous experience matters remains an open question. Food quality may well have had an influence in earlier phases of this work. We now avoid crowding in the larval cultures.

A full account of the different components of interindividual variance in visual learning at the flight simulator would be a major enterprise exceeding by far the scope of this study. First, one would have to ask how much of the variance is experimentally induced, how much is due to the fluctuations of the behavior in each animal (intraindividual variance), and how much reflects true interindividual differences (i.e., differences between individuals that are to some extent timeinvariant). We have tested the possibility of exper- imentally induced variation by deliberately changing some of the manipulations such as the positioning of the hook on the fly's thorax, the relative position of the head to the body, the duration of cold anesthesia, and the precise orientation in the flight simulator. These parameters seem not to be critical once the experimenter has acquired the basic skills in handling the flies and the apparatus. Most of the variance in learning indices seems to be either intraindividual or interindividual.

The fluctuations in learning performance of the individual fly have not been considered here but constitute a major part of the total variance ( $R$. Ernst, B. Brembs, R. Wolf, and M. Heisenberg, in prep.). Visual orientation at the flight simulator is a highly artificial behavior. The simulation concerns but a single degree of freedom: rotation around the vertical body axis. Even for this one variable, compromises in the dynamics of the artificial feedback are unavoidable (Heisenberg and Wolf 1993). Flies may spend much of the time trying to escape from this bizarre situation. Hence, part of the fluctuations may reflect a labile balance between diverging goals: heat avoidance and escape from the hook. Moreover, the heat conditioning itself is totally unrealistic. The distribution of hot and cold regions in the fly's virtual space cannot be described in Cartesian coordinates. Wherever the fly happens to be, it is infinitely close to a temperature boundary, and it can get from one temperature zone to another just by turning. In such a confusing situation the fly cannot be expected to perform consistently all the time. It would not be surprising if much of the variability in learning performance were a consequence of the special experimental design. On the other hand, heat avoidance conditioning of walking flies in a little box seems to be as variable (Wustmann et al. 1996).

A further potential source of intraindividual variance is the test situation. While learning indices are recorded, flies may learn that the nonpermissive flight orientations are no longer associated with heat (extinction). This learning is an operant behavior and therefore a stochastic process. In other words, some flies are quick, others take longer in adjusting to the new situation (Wolf and Heisenberg 1991).

In this study we are concerned only with the interindividual variance and with only three of its components: age, experience in the flight simulator, and nutrition. There are several other obvious reasons why flies of a regular Drosopbila labora-

$$
\text { , n....... }
$$


tory culture may differ from each other. One is the genetic heterogeneity. In our wild-type cultures $\sim 100$ individuals are transferred from one bottle to the next. Hence, flies are far from isogenic. Another is the variation in the developmental process (amplified molecular "noise"), of which we have no control. Although all may contribute significantly, it is difficult to estimate how much.

Our finding that 1 - to 2 -day-old flies learn less well than 3- to 4-day-old ones is unexpected since in conditioned courtship suppression and in olfactory learning 2-day-old flies are fully competent (Tully and Quinn 1985). We conclude that in Drosopbila melanogaster some aspects of visual orientation behavior as assessed in the flight simulator are not fully matured until four days after eclosion from the pupa. Behavioral maturation at this late age also has been reported recently by Hirsch et al. (1990, 1995; Barth et al. 1996), who observed that dark rearing affects the flies' behavior in a visual orientation task and in a courtship competition assay and that these differences are apparent after 4 but not yet after 2 days of dark rearing. Learning indices in conditioned visual orientation in the flight simulator are affected by a large number of behavioral parameters and may not directly reflect synaptic plasticity. Synaptic plasticity may be developed in the visual system as early as in the olfactory system. The late maturation may occur in some other aspect of visual orientation behavior that is required for the performance.

A second factor contributing to the differences among flies may be the experience a fly has gathered prior to the experiment. This parameter is difficult to control. We have demonstrated here that practice in the flight simulator has a pronounced effect on learning performance. Dill et al. (1995) have shown that even without reinforcement visual learning occurs in the flight simulator, and earlier work suggests that also motor learning may continuously take place at the torque meter (Wolf and Heisenberg 1991; Weidtmann 1993). It is well known from learning studies with other animals that familiarity with the general situation of the conditioning experiment may reduce variability in the performance and may thus have a positive influence on the learning index (e.g., $\mathrm{Ng}$ et al. 1991). It is not clear from our result whether uncontrolled experiences during the 2-4 days before the experiment contribute to the variance in the standard experiment.

The most dramatic finding is the amnesic ef- fect of the Beijing fly food. Even with a recipe that is highly nutritious, it may well happen in the normal maintenance routine that for several generations in a row, flies have severely depleted food. This is likely to occur if flies are transferred to fresh bottles over large intervals. Those flies will be unable to learn even if transferred to a rich food source before the experiment. This effect may contribute heavily to the variability unless optimal growth conditions are provided for the larvae over the generations. The finding that the fly food may have an influence on learning is not entirely new. Tully et al. (1994) have tried several recipes that produced lower learning indices than the one they used routinely. It is not obvious from their report how many generations of flies were kept on the alternative media. More dramatic effects might have been obtained with longer exposures.

Gradual changes in behavior over several generations after a dramatic event such as a radical change in food quality might be explained by natural selection in the food bottles. However, the experiments are not performed with natural populations but with continuously inbred laboratory stocks. Moreover, in the case of shifts from Beijing to Würzburg food the flies had been subjected to the inverse shift (and thus to a putative selection process in the opposite direction) a few generations before. An additional difficulty with this line of reasoning is that it does not explain why the putative selection would disfavor learning ability. Perhaps starvation selects for those genetic variants that can channel all their nutritional resources into egg production, even at the expense of learning ability.

Quite a different explanation for the above finding would be to assume that austere nutritional conditions have a low probability of triggering a discrete variant of larval development leading to flies without learning ability. In many species environmental factors trigger different developmental programs. Conspicuous examples are the caste systems of social insects and sexual differentiation of reptiles. Recently, D. Emlen (in prep.) has reported that food quality determines the ratio of horned and hornless individuals in populations of the scarab beetle Ontbophagus acuminatus. In our case one would have to assume further that the two developmental programs leading to the two "morphs" (i.e., learners or nonlearners) would be transmitted to the next generation (for instance, the morph of the female might determine the morph of the offspring).

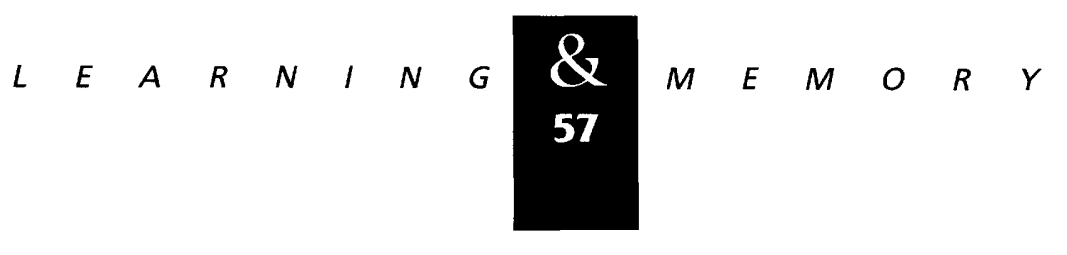


Cumulative environmental effects over several generations in Drosophila melanogaster are not without precedence. Ho et al. (1983) elicited phenocopies of bitborax after treatment with ether and observed that the penetrance and expression of these phenocopies increased in successive generations. They ruled out selection and offered "cumulative cytoplasmic modification" as the most likely explanation. We do not know what that might be in molecular terms, but we would like to emphasize that at present no plausible explanation can be offered for the gradual loss (and gain) of learning ability. It will be important to know whether the switch from Beijing to Würzburg food is indeed slower than the switch in the other direction; whether Beijing food contains an inhibitor of learning or lacks a requirement for it; whether the learning defect is transmitted via females, males, or both sexes; and whether malnutrition affects all kinds of learning or just conditioned visual flight orientation. A system of morphs, learners, and nonlearners in Drosophila would have the interesting implications that, from an evolutionary perspective, learning and memory may be dispensable in certain environments.

\section{Acknowledgments}

We would like to thank K.G. Götz, Tübingen, for substantial support in setting up the flight simulator in Beijing and for invaluable suggestions and discussions. R. Ernst kindly provided data for Figure 1. We appreciate the many suggestions of one of the reviewers. This work was made possible by a cooperation agreement between the National Science Foundation of China and the German Science Foundation and by a donation of equipment from the Max Planck Society.

The publication costs of this article were defrayed in part by payment of page charges. This article must therefore be hereby marked "advertisement" in accordance with 18 USC section 1734 solely to indicate this fact.

\section{References}

Aceves-Pina, E.O., R. Booker, J.S. Duerr, M.S. Livingstone, W.G. Quinn, R.F. Smith, P.P. Sziber, B.L. Tempel, and T.P. Tully. 1983. Learning and memory in Drosophila, studies with mutants. Cold Spring Harbor Symp. Quant. Biol. 48: 831-839.

Barth, M., H.V.B. Hirsch, and M. Heisenberg. 1996. Rearing in different light regimes leads to positive assortative mating in Drosophila melanogaster. Anim. Behav. (in press).

Davis, R.L. 1993. Mushroom bodies and Drosophila learning. Neuron 11: 1-14.

DeZazzo, J. and T. Tully. 1995. Dissection of memory formation: From behavioral pharmacology to molecular genetics. Trends Neurosci. 18: 212-218.

Dill, M. 1995. "Unterscheidung und Wiedererkennung visueller Muster bei der Taufliege Drosophila melanogaster." Ph.D. thesis. Würzburg University, Würzburg, Germany.

Dill, M. and M. Heisenberg. 1995. Visual pattern memory without shape recognition. Phil. Trans. R. Soc. Lond. B 349: 143-152.

Dill, M., R. Wolf, and M. Heisenberg. 1993. Visual pattern recognition in Drosophila involves retinotopic matching. Nature 365: 751-753.

1995. Behavioral analysis of Drosophila landmark learning in the flight simulator. Learning \& Memory 2: $152-160$

Dudai, Y. 1988. Neurogenetic dissection of learning and short-term memory in Drosophila. Ann. Rev. Neurosci. 11: $537-563$.

Heisenberg, M. 1989. Genetic approach to learning and memory (mnemogenetics) in Drosophila melanogaster. In Fundamentals of memory formation: Neuronal plasticity and brain function (ed. H. Rahmann), pp. 3-45. Gustav Fischer Verlag, Stuttgart, Germany.

Heisenberg, M. and R. Wolf. 1984. Vision in Drosophila: Genetics of microbehavior (ed. V. Braitenberg). Springer, New York, NY.

1988. Reafferent control of optomotor yaw torque in Drosophila melanogaster. I. Comp. Physiol. A 163: 373-388.

1993. The sensory motor link in motion-dependent flight control of flies. In Visual motion and its role in the stabilization of gaze (ed. F.A. Miles and J. Wallman), pp. 265-283. Elsevier, Amsterdam, The Netherlands.

Heisenberg, M., A. Borst S. Wagner, and D. Byers. 1985. Drosophila mushroom body mutants are deficient in olfactory learning. /. Neurogenet. 2: 1-30.

Heisenberg, M., M. Heusipp, and, C. Wanke. 1995. Structural plasticity in the Drosophila brain. I. Neurosci. 15: 1951-1960.

Hirsch, H.V.B., D. Potter, D. Zawierucha, T. Choudhri, A. Glasser, R.K. Murphy, and D. Byers. 1990. Rearing in darkess changes visually-guided choice behavior in Drosophila. Visual Neurosci. 5: 281-289.

Hirsch, H.V.B., M. Barth, S. Luo, H. Sambaziotis, M. Huber, D. Possidente, H. Ghiradella, and L. Tompkins. 1995. Early visual experience affects mate choice of Drosophila melanogaster. Anim. Behav. 50: 1211-1217.

Ho, M.W., C. Tucker, D. Keely, and P.T. Saunders. 1983. Effects of successive generations of ether treatment on penetrance and expression of the bithorax phenocopy in Drosophila melanogaster. J. Exp. Zool. 225: 357-368.

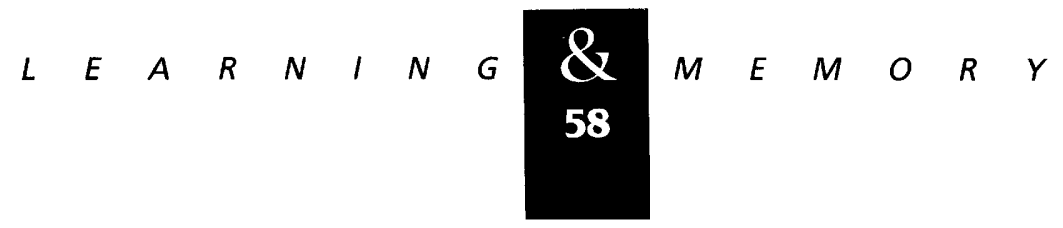


Kandel, E. and T. Abel. 1995. Neuropeptides, adenylyl cyclase, and memory storage. Science 268: 825-826.

Ng, K.T., M.E. Gibbs, and S. Crowe. 1991. La Trobe procedure. In Neuronal and behavioral plasticity (ed. R.J. Andrew), pp. 18-27. Oxford University Press, New York, NY.

Quinn, W.G., W.A. Harris, and S. Benzer. 1974.

Conditioned behavior in Drosophila melanogaster. Proc. Natl. Acad. Sci. 71: 708-712.

Sachs, L. 1992. Angewandte Statistik. Springer, Heidelberg, Germany.

Tompkins, L., R.W. Siegel, D.A. Gailey, and ).C. Hall. 1983. Conditioned courtship in Drosophila and its mediation by association of chemical cues. Behav. Genet. 13: 565-578

Tully, T. and W.G. Quinn. 1985. Classical conditioning and retention in normal and mutant Drosophila melanogaster. I. Comp. Physiol. A 156: 263-277.

Tully, T., T. Preat, S.C. Boynton, and M. Del Vecchio. 1994. Genetic dissection of consolidated memory in Drosophila. Cell 79: 35-47.

Weidtmann, N. 1993. "Visuelle Flugsteuerung und Verhaltensplastizität bei Zentralkomplex-Mutanten von Drosophila melanogaster." Diplom thesis. Würzburg University, Würzburg, Germany.

Wolf, R. and M. Heisenberg. 1991. Basic organization of operant behavior as revealed in Drosophila flight orientation. I. Comp. Physiol. A 169: 699-705.

Wustmann, G., K. Rein, R. Wolf, and M. Heisenberg. 1996. A new paradigm for operant conditioning of Drosophila melanogaster. I. Comp. Physiol. A (in press).

Received May 28, 1996; accepted in revised form July 26, 1996. 


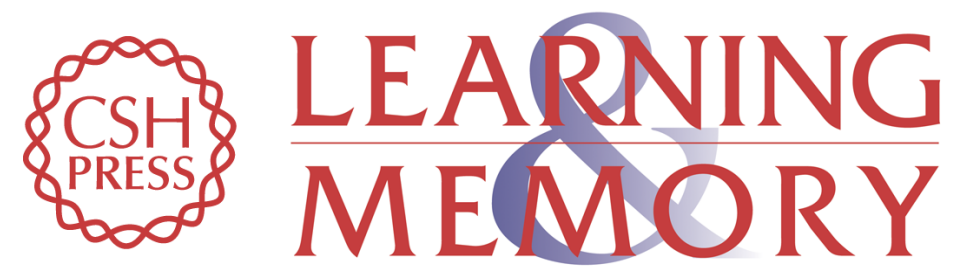

\section{Conditioned visual flight orientation in Drosophila: dependence on age, practice, and diet.}

A Guo, L Li, S Z Xia, et al.

Learn. Mem. 1996, 3:

Access the most recent version at doi:10.1101//m.3.1.49

References This article cites 19 articles, 5 of which can be accessed free at: http://learnmem.cshlp.org/content/3/1/49.full.html\#ref-list-1

License

Email Alerting Receive free email alerts when new articles cite this article - sign up in the box at the Service top right corner of the article or click here. 